\title{
BMJ Open Chronic hepatitis B virus infection and total and cause-specific mortality: a prospective cohort study of 0.5 million people
}

\author{
Jiahui $\mathrm{Si},{ }^{1}$ Canqing Yu, ${ }^{1}$ Yu Guo, ${ }^{2}$ Zheng Bian, ${ }^{2}$ Ruogu Meng, ${ }^{1}$ Ling Yang, ${ }^{3}$ \\ Yiping Chen, ${ }^{3}$ Jianrong Jin, ${ }^{4}$ Jingchao Liu, ${ }^{4}$ Ziyan Guo, ${ }^{5}$ Junshi Chen, ${ }^{6}$ \\ Zhengming Chen, ${ }^{3}$ Jun Lv, ${ }^{1,7}$ Liming Li, ${ }^{1,2}$ on behalf of the China Kadoorie Biobank \\ Collaborative Group
}

To cite: Si J, Yu C, Guo Y, et al. Chronic hepatitis B virus infection and total and cause-specific mortality: a prospective cohort study of 0.5 million people. BMJ Open 2019;0:e27696. doi:10.1136/ bmjopen-2018-027696

- Prepublication history and additional material for this paper are available online. To view these files, please visit the journal online (http://dx.doi. org/10.1136/bmjopen-2018027696).

Received 6 November 2018 Revised 13 January 2019 Accepted 16 January 2019

Check for updates

(C) Author(s) (or their employer(s)) 2019. Re-use permitted under CC BY-NC. No commercial re-use. See rights and permissions. Published by BMJ.

For numbered affiliations see end of article.

\section{Correspondence to}

Dr Liming Li;

Imlee@vip.163.com and

Dr Jun Lv;

Ivjun@bjmu.edu.cn

\section{ABSTRACT}

Objectives Chronic hepatitis B virus (HBV) infection is associated with a higher risk of liver diseases. Substantial uncertainty remains, however, about the associations of HBV infection with mortality from extrahepatic causes, especially from subtypes of cardiovascular diseases. We prospectively examined the association of chronic HBV infection with total and cause-specific mortality. Design Population-based prospective cohort study. Setting China Kadoorie Biobank in which participants from 10 geographically diverse areas across China were enrolled between 2004 and 2008.

Participants 475801 participants 30-79 years of age without reporting major chronic diseases at baseline were enrolled. Hepatitis B surface antigen ( $\mathrm{HBsAg}$ ) was tested using an on-site rapid test strip at baseline.

Primary and secondary outcome measures Total and cause-specific mortality.

Results A total of 35822 deaths were recorded during $\sim 10$ years of follow-up. In multivariable-adjusted analyses, compared with HBsAg-negative participants, HBsAg-positive participants had an increased risk of total mortality ( $\mathrm{HR}=2.01,95 \% \mathrm{Cl}: 1.91$ to 2.12), which was higher in men ( $\mathrm{HR}=2.16,95 \% \mathrm{Cl}: 2.01$ to 2.31 ) than in women ( $\mathrm{HR}=1.74,95 \% \mathrm{Cl}: 1.60$ to 1.90$)$. Presence of HBsAg was associated with increased mortality from liver cancer (1339 deaths, $\mathrm{HR}=13.95,95 \% \mathrm{Cl}: 12.46$ to 15.62 ), infections ( 410 deaths, $\mathrm{HR}=10.30,95 \% \mathrm{Cl}: 8.21$ to 12.94 ), digestive diseases ( 688 deaths, $\mathrm{HR}=6.83,95 \% \mathrm{Cl}: 5.49$ to 8.50), intracerebral haemorrhage (4077 deaths, $\mathrm{HR}=1.38$, $95 \% \mathrm{Cl}: 1.14$ to 1.68 ) and ischaemic heart diseases (4624 deaths, $\mathrm{HR}=1.31,95 \% \mathrm{Cl}: 1.09$ to 1.58 ). The positive association between $\mathrm{HBsAg}$ status and risk of death was stronger in participants younger than 50 years, smokers, physically active or non-hypertensive participants. Conclusions Among Chinese adults, chronic HBV infection was associated with increased mortality from a range of hepatic and extrahepatic diseases.

\section{INTRODUCTION}

Hepatitis B virus (HBV) infection is a major health problem worldwide. In 2016, the

\section{Strengths and limitations of this study}

- In this Chinese population, besides the significantly increased risk of death from liver cancer, infections and digestive diseases, we observed an increased risk of death due to intracerebral haemorrhage and ischaemic heart diseases associated with chronic hepatitis B virus (HBV) infection.

- The results highlight the importance of health advice on quitting smoking and early screening for major chronic diseases in people with chronic HBV infection.

- The prospective cohort design, large sample size and extended follow-up period allowed the present study to produce reliable risk estimates for total and various cause-specific mortality.

- We used an on-site hepatitis B surface antigen (HBsAg) rapid test that is convenient and repeatable but with low sensitivity for the lower sero-HBsAg level.

- Lack of information about specific treatment and other serologic markers of HBV infection also led to limitations of the present study.

worldwide estimated prevalence of chronic HBV infection was $3.5 \%$, and 257 million have chronic infection. ${ }^{12}$ In 2015, an estimated 887000 deaths worldwide occurred due to HBV infection. ${ }^{3}$ The prevalence of HBV infection in China has changed from highly endemic to intermediate endemic in the past two decades. However, China still had the greatest number of people living with HBV infection, ${ }^{45}$ with a total of 100 million infected. ${ }^{6}$

The long-term sequelae of chronic HBV infection have been well documented in terms of its effects on the liver. ${ }^{7}$ Also, HBV causes systemic effects and immune response and may colonise in the vascular tissues, ${ }^{8}$ which suggests that HBV-induced extrahepatic 
manifestations may exist. However, conflicting results have been reported on the association between HBV infection and risk of death due to extrahepatic outcomes like cardiovascular and respiratory diseases. ${ }^{9-18}$ Most of the previous studies were conducted in Western populations with a relatively low prevalence of $\mathrm{HBV}$ infection. These studies were usually based on a specialised population-based registry and did not adjust for potential confounders due to limited information available. ${ }^{9-14} \mathrm{Few}$ prospective studies conducted in Chinese population ${ }^{15-18}$ were characterised by small sample size, single-gender participants or population of a particular geographical area. Most of these studies did not find an association of HBV infection with risk of death due to cardiovascular and respiratory diseases.

Nowadays, a considerable amount of Chinese adults are suffering from chronic HBV infection and its sequelae, but the full impact on mortality from both hepatic and extrahepatic causes is still less well established. This nationwide study including both rural and urban residents prospectively examined the association of chronic HBV infection with cause-specific mortality, especially deaths from extrahepatic causes in a large cohort of 0.5 million adult Chinese. We additionally investigated potential impact of baseline lifestyle factors and prevalent diseases on the association between HBV infection and death.

\section{METHODS}

\section{Study population}

The China Kadoorie Biobank (CKB) cohort was established in 10 geographically diverse areas across China, 5 urban sites (in Harbin, Qingdao, Suzhou, Liuzhou and Haikou) and 5 rural sites (in Henan, Gansu, Sichuan, Zhejiang and Hunan) during 2004-2008. In each area, all non-disabled, permanent residents aged $35-74$ years were invited to participate in the study. To encourage participation, a few participants who were slightly outside the targeted age range were not turned away. Of the total of $\sim 1.8$ million eligible adults in these areas, almost one in three responded. A total of 512891 participants were enrolled in the cohort. All participants had valid baseline data, including the completed questionnaire, physical measurements and written informed consent. Further details of the CKB study have been given elsewhere. ${ }^{19} 20$

After correction for errors in age and exclusion of participants with age outside of the 30-79 years, 512713 participants were eligible for inclusion in the study. In the present analysis, we excluded participants who reported having stroke $(n=8884)$, heart disease $(n=15472)$ or cancer $(\mathrm{n}=2577)$; had missing data or unclear result for hepatitis B surface antigen (HBsAg) $(\mathrm{n}=11396)$; had missing data for body mass index (BMI) $(n=2)$ or were lost to follow-up shortly after baseline $(n=1)$. We finally had 475801 participants in the analyses.

\section{Assessment of exposure}

At baseline, the whole venous blood of participants was applied to an on-site rapid test strip (ACON dipstick). The qualitative test result for HBsAg status included negative, positive or unclear. All participants also reported if they had ever been diagnosed with chronic hepatitis (not limited to chronic viral hepatitis) or liver cirrhosis (yes or no) by a qualified doctor using laptop-based questionnaires. For those who reported the disease, we further asked their age at initial diagnosis, and if they were still on treatment (yes or no).

\section{Assessment of covariates}

For each participant, detailed covariate information was collected by means of a structured questionnaire at baseline, including sociodemographic characteristics (age, sex, education, occupation, household income and marital status), lifestyle behaviours (alcohol consumption, tobacco smoking, physical activity and intakes of fresh vegetables, fruit and red meat), personal health and medical history (hypertension, diabetes and women's menopause status), and information on family histories of cancer, heart attack and stroke. To avoid misleadingly elevated risk, we included former smokers who had stopped smoking for illness in the current smoker category. The daily level of physical activity was calculated by multiplying the metabolic equivalent task (MET) value for a particular type of activity by hours spent on that activity per day, and then summing the MET-hours for all activities. The family history of a particular disease was defined if participants reported at least one first degree relative with the disease.

Trained staff measured weight, height, the circumference of waist and hip and blood pressure with calibrated instruments. BMI was calculated as weight in kilograms divided by the square of the height in metres. The waisthip ratio (WHR) was the ratio of waist circumference to hip circumference. Prevalent hypertension was defined as measured systolic blood pressure $\geq 140 \mathrm{~mm} \mathrm{Hg}$, measured diastolic blood pressure $\geq 90 \mathrm{~mm} \mathrm{Hg}$, a self-reported prior diagnosis of hypertension or self-reported use of antihypertensive medication at baseline. Prevalent diabetes was defined as measured fasting blood glucose $\geq 7.0 \mathrm{mmol} / \mathrm{L}$, measured random blood glucose $\geq 11.1 \mathrm{mmol} / \mathrm{L}$ or a self-reported prior diagnosis of diabetes at baseline.

\section{Ascertainment of deaths}

We ascertained vital status using linkage with local death registries $^{21}$ and residential records. The electronic linkage to the national health insurance claim databases started in 2011 and has been achieved for about $97 \%$ of participants in 2014, which has become an important way of follow-up. For participants who failed to be linked, trained staff would actively follow them to ascertain their status annually. The causes of death were sought mainly from official death certificates that were supplemented, if necessary, by reviewing medical records or undertaking a 
verbal autopsy using validated instruments for those with unknown cause of death reported.

Trained staff blinded to baseline information coded all death using the International Classification of Diseases, Tenth Revision (ICD-10). Scanned images of the death certificates were further reviewed centrally. The deaths were then categorised into nine groups: ischaemic heart diseases (I20-I25), cerebral infarction (I63), intracerebral haemorrhage (I61), liver and intrahepatic bile duct cancer (C22, liver cancer, for short), other cancers (C00-C97 except C22), diseases of the respiratory system (J00-J99), diseases of the digestive system (K00-K93), infections (A00-B99) and all other causes.

\section{Statistical analysis}

Person-years at risk were calculated for each participant from baseline to the date of death, loss to follow-up or 31 December 2016, whichever came first. The Cox proportional hazards model was used to estimate the HR and $95 \% \mathrm{CI}$, with age as the underlying time scale, and stratified jointly by 5 -year age groups and 10 survey sites.

Multivariable models were adjusted for established and potential risk factors for death: sex (men or women, for whole cohort), level of education (no formal school, primary school, middle school, high school, college, or university or higher), marital status (married, widowed, divorced or separated, or never married), alcohol consumption (less than weekly drinker, weekly drinker, daily drinker with an intake of $<15,15-29,30-59$ or $\geq 60 \mathrm{~g} /$ day), smoking status (non-smoker, former smoker having quit smoking $\geq 5$ or $<5$ years previously or current smoker smoked $<15,15-24$ or $\geq 25$ cigarettes or equivalents per day), physical activity (MET-hours/day), frequencies of fresh vegetable, fruit and red meat intake (daily, 4-6 days/week, 1-3 days/week, monthly, or rarely or never), menopausal status (for women only), status of family history of heart attack, stroke or cancer (presence or absence, adjusted for only in the corresponding analysis of cause-specific mortality), BMI $\left(\mathrm{kg} / \mathrm{m}^{2}\right)$, WHR (continuous variable) and prevalent diabetes and hypertension (presence or absence). To further explore whether the association between chronic HBV infection and risk of mortality was mediated or confounded by the presence of chronic hepatitis or cirrhosis, we additionally adjusted for a composite variable of disease status (absence or presence), duration ( $<15$ or $\geq 15$ years) and treatment status at baseline (no or yes). We also examined the joint associations of HBsAg status and the presence of chronic hepatitis or cirrhosis with total and cause-specific mortality. To examine the robustness of the findings, we conducted several sensitivity analyses: excluding participants who died during the first 2 years of follow-up, or further adjusting for occupation (agriculture and related, factory, administrator or manager, professional or technical, sales or service, retired, housewife, self-employed, unemployed or others) and household income $(<2500$, 2500-4999, 5000-9999, 10000-19,999, 20000-34,999 or $\geq 35000 ¥ /$ year).
Age at death between HBsAg-positive and HBsAg negative participants was compared using Wilcoxon rank-sum test. We examined the association of HBsAg status with total mortality among prespecified baseline subgroups based on sex (men or women), age at baseline $(<50,50-59$ or $\geq 60$ years), residence (urban or rural), smoking status (daily smoker or not), alcohol consumption (daily drinker or not), level of physical activity (categorised using tertile cutoffs), BMI ( $<24.0$ or $\left.\geq 24.0 \mathrm{~kg} / \mathrm{m}^{2}\right)$, prevalent diabetes (presence or absence) and prevalent hypertension (presence or absence). The tests for interaction were performed by means of likelihood ratio test comparing models with and without cross-product term.

The statistical analyses were performed with Stata V.14.2 (StataCorp). Statistical significance was set at two-tailed $\mathrm{p}<0.05$. P values were presented as unadjusted for multiple testing unless otherwise indicated. For testing of multiple primary outcomes, we applied Bonferroni correction to the significance level, dividing 0.05 by 10 outcomes examined (ie, 0.005).

\section{Patient and public involvement}

Patients were not involved in the present study. The results of the main study were presented to study participants at the website of the CKB study (http://www.ckbiobank.org/site/) and by newsletters annually.

\section{RESULTS}

The mean age of 475801 participants was $51.6 \pm 10.6$ years. Men and rural residents accounted for $41.0 \%$ and $56.4 \%$ of participants, respectively. HBsAg-positive participants were more likely to be younger, men and urban residents, especially in those with chronic hepatitis or cirrhosis (table 1). Among participants who reported a medical history of chronic hepatitis or cirrhosis, HBsAg-positive participants had a shorter duration of the disease than HBsAg-negative ones.

A total of 35822 deaths (20456 men and 15366 women) and 4935 participants lost to follow-up were documented over 4.7 million person-years of follow-up (median follow-up: 10.1 years; IQR: 1.88 years). The crude mortality rates were 7.08 deaths per 1000 personyears for all causes, 1.01 for ischaemic heart diseases, 0.30 for cerebral infarction, 0.89 for intracerebral haemorrhage, 0.29 for liver cancer, 2.15 for other cancers, 0.74 for respiratory diseases, 0.15 for digestive diseases, 0.09 for infections and 1.85 for all other causes.

After adjustment for established and potential risk factors for death, HBsAg positivity was associated with increased risks of death due to ischaemic heart diseases (HR=1.31, 95\% CI: 1.09 to 1.58$)$ and intracerebral haemorrhage (HR=1.38, 95\% CI: 1.14 to 1.68 ). Also, we found strong positive associations of HBsAg positivity with liver cancer (HR=13.95, 95\% CI: 12.46 to 15.62), infections $(\mathrm{HR}=10.30$, 95\% CI: 8.21 to 12.94$)$ and digestive diseases $(\mathrm{HR}=6.83,95 \%$ CI: 5.49 to 8.50$)$ (table 2). No statistically significant association was observed for cerebral 
Table 1 Baseline characteristics according to HBsAg status and prevalent chronic hepatitis and cirrhosis at baseline among 475801 participants

\begin{tabular}{|c|c|c|c|c|}
\hline \multirow[b]{2}{*}{ Characteristics } & \multicolumn{2}{|l|}{ HBsAg negative } & \multicolumn{2}{|l|}{ HBsAg positive } \\
\hline & $\begin{array}{l}\text { Without hepatitis or } \\
\text { cirrhosis }\end{array}$ & $\begin{array}{l}\text { With hepatitis or } \\
\text { cirrhosis }\end{array}$ & $\begin{array}{l}\text { Without hepatitis or } \\
\text { cirrhosis }\end{array}$ & $\begin{array}{l}\text { With hepatitis or } \\
\text { cirrhosis }\end{array}$ \\
\hline No of participants & 456975 & 3829 & 13317 & 1680 \\
\hline Age (years) & 51.6 & 52.1 & 49.4 & 46.7 \\
\hline Men (\%) & 40.5 & 59.4 & 45.0 & 61.2 \\
\hline Rural area (\%) & 56.6 & 60.9 & 48.8 & 48.2 \\
\hline Married (\%) & 90.9 & 91.3 & 90.3 & 89.9 \\
\hline Middle school and higher (\%) & 49.6 & 49.1 & 47.2 & 51.2 \\
\hline \multicolumn{5}{|l|}{ Daily tobacco smoker (\%) } \\
\hline Men & 67.7 & 65.2 & 69.0 & 65.1 \\
\hline Women & 2.7 & 2.7 & 3.1 & 2.3 \\
\hline \multicolumn{5}{|l|}{ Daily alcohol drinker (\%) } \\
\hline Men & 20.9 & 15.6 & 22.0 & 9.7 \\
\hline Women & 0.9 & 0.7 & 1.2 & 0.8 \\
\hline Physical activity (MET-hours/day) & 21.5 & 20.2 & 21.6 & 19.6 \\
\hline \multicolumn{5}{|l|}{ Weekly consumption* } \\
\hline Red meat (days) & 3.7 & 3.7 & 3.7 & 3.7 \\
\hline Fresh vegetables (days) & 6.8 & 6.8 & 6.8 & 6.8 \\
\hline Fresh fruits (days) & 2.5 & 2.7 & 2.5 & 2.8 \\
\hline Body mass index $\left(\mathrm{kg} / \mathrm{m}^{2}\right)$ & 23.6 & 23.5 & 23.5 & 23.5 \\
\hline Postmenopausal (\%)† & 50.8 & 52.5 & 50.9 & 51.0 \\
\hline Prevalent diabetes (\%) & 5.4 & 6.2 & 5.7 & 6.3 \\
\hline Prevalent hypertension (\%) & 33.9 & 30.8 & 32.7 & 28.7 \\
\hline Duration of the disease (years) $\ddagger$ & - & 17.7 & - & 13.0 \\
\hline Under treatment at baseline (\%)‡ & - & 10.5 & - & 19.2 \\
\hline
\end{tabular}

All variables were adjusted for age, sex and survey sites, as appropriate. We observed the statistically significant differences in all baseline characteristics between HBsAg-negative and HBsAg-positive groups, except for smoking in women $(p=0.056)$ and prevalent diabetes $(p=0.167)$.

*Weekly consumptions of red meat, fresh vegetables and fruits were calculated by assigning participants to the midpoint of their consumption category.

†Among 281244 female participants.

$\ddagger$ Among participants who had prevalent chronic hepatitis or liver cirrhosis at baseline.

HBsAg, hepatitis B surface antigen; MET, metabolic equivalent of task.

infarction, other cancers except for liver cancer and respiratory diseases in the whole cohort. The association between HBsAg status and liver cancer was stronger in men $(\mathrm{HR}=15.05$, 95\% CI: 13.12 to 17.27$)$ than in women $(\mathrm{HR}=10.39,95 \%$ CI: 8.36 to 12.91$) \quad\left(\mathrm{p}_{\text {interaction }}<0.001\right)$ (see online supplementary files 1 and 2). Other associations were consistent across sex (all $\mathrm{p}_{\text {interaction }}>0.05$ ).

In total, HBsAg positivity was significantly associated with an increased risk of total mortality (table 2). In all eligible participants, compared with HBsAg-negative participants, the HR (95\% CI) for death was 2.01 (1.91 to 2.12) for HBsAg-positive participants. The association between HBsAg status and total mortality was stronger in men (HR=2.16, 95\% CI: 2.01 to 2.31$)$ than in women
(HR=1.74, 95\% CI: 1.60 to 1.90 ) (see online supplementary files 1 and 2; $\mathrm{p}_{\text {interaction }}<0.001$ ).

Further adjustment for the presence of chronic hepatitis or cirrhosis at baseline only slightly attenuated the associations (table 2). Further adjustment for occupation and household income did not change the risk estimates appreciably (see online supplementary file 3 ). We also excluded participants who died during the first 2 years of follow-up, the risk estimates largely remained (see online supplementary file 4).

The median age at death was significantly younger for 7.7 years (men: 8.7 years; women: 5.5 years) among HBsAg-positive participants than among HBsAg-negative ones $(p<0.001)$. Younger median age at death associated 


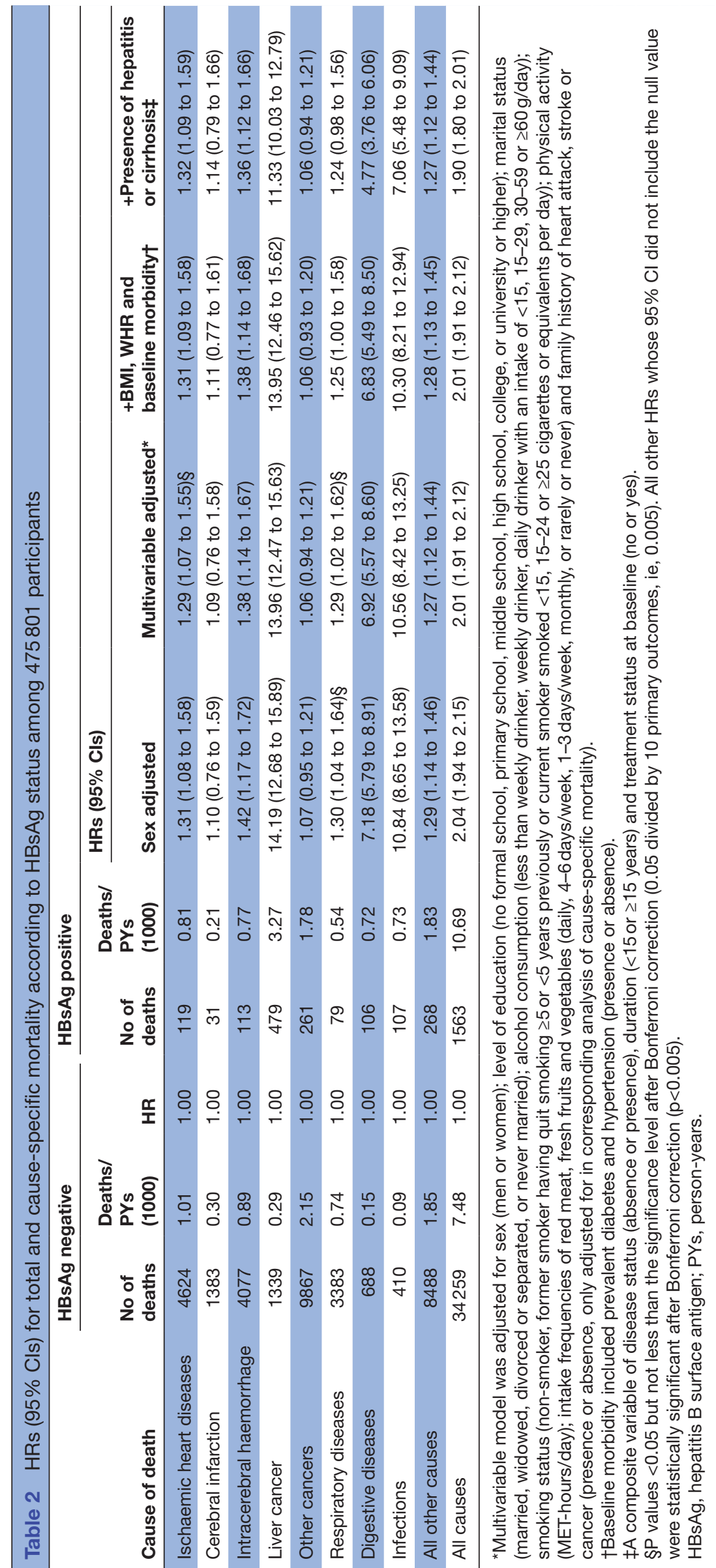


Open access

Table 3 HRs (95\% Cls) for total and cause-specific mortality according to HBsAg status and presence of chronic hepatitis or cirrhosis at baseline among 475801 participants

\begin{tabular}{|c|c|c|c|c|}
\hline \multirow[b]{2}{*}{ Cause of death } & \multicolumn{2}{|l|}{ HBsAg negative } & \multicolumn{2}{|l|}{ HBsAg positive } \\
\hline & $\begin{array}{l}\text { Without hepatitis or } \\
\text { cirrhosis }\end{array}$ & With hepatitis or cirrhosis & Without hepatitis or cirrhosis & With hepatitis or cirrhosis \\
\hline No of deaths & 4591 & 33 & 111 & 8 \\
\hline Deaths/PYs (1000) & 1.01 & 0.88 & 0.85 & 0.50 \\
\hline HRs (95\% Cls) & 1.00 & 0.84 (0.59 to 1.19$)$ & 1.31 (1.08 to 1.60$)$ & 1.24 (0.62 to 2.50$)$ \\
\hline No of deaths & 1376 & 7 & 28 & 3 \\
\hline Deaths/PYs (1000) & 0.30 & 0.19 & 0.21 & 0.19 \\
\hline HRs (95\% Cls) & 1.00 & $0.58(0.27$ to 1.22$)$ & 1.07 (0.73 to 1.58$)$ & 1.54 (0.48 to 4.88$)$ \\
\hline \multicolumn{5}{|c|}{ Intracerebral haemorrhage } \\
\hline No of deaths & 4047 & 30 & 103 & 10 \\
\hline No of deaths & 1261 & 78 & 383 & 96 \\
\hline Deaths/PYs (1000) & 0.28 & 2.09 & 2.94 & 6.02 \\
\hline HRs (95\% Cls) & 1.00 & 6.57 (5.16 to 8.36$)$ & $13.00(11.50$ to 14.71$)$ & 31.33 (24.84 to 39.51$)$ \\
\hline \multicolumn{5}{|l|}{ Other cancers } \\
\hline No of deaths & 9777 & 90 & 238 & 23 \\
\hline Deaths/PYs (1000) & 2.15 & 2.41 & 1.83 & 1.44 \\
\hline HRs (95\% Cls) & 1.00 & $0.90(0.73$ to 1.11$)$ & $1.06(0.93$ to 1.21$)$ & 1.05 (0.69 to 1.59$)$ \\
\hline \multicolumn{5}{|l|}{ Respiratory diseases } \\
\hline No of deaths & 3344 & 39 & 72 & 7 \\
\hline No of deaths & 373 & 37 & 81 & 26 \\
\hline Deaths/PYs (1000) & 0.08 & 0.99 & 0.62 & 1.63 \\
\hline HRs (95\% Cls) & 1.00 & 11.67 (8.17 to 16.67$)$ & 9.48 (7.35 to 12.22$)$ & 31.72 (20.33 to 49.49$)$ \\
\hline \multicolumn{5}{|l|}{ All other causes } \\
\hline No of deaths & 8417 & 71 & 238 & 30 \\
\hline Deaths/PYs (1000) & 1.85 & 1.90 & 1.83 & 1.88 \\
\hline HRs (95\% Cls) & 1.00 & $0.90(0.71$ to 1.14$)$ & $1.23(1.08$ to 1.41$)$ & 1.77 (1.23 to 2.55$)$ \\
\hline \multicolumn{5}{|l|}{ All causes } \\
\hline No of deaths & 33829 & 430 & 1330 & 233 \\
\hline Deaths/PYs (1000) & 7.44 & 11.50 & 10.20 & 14.62 \\
\hline HRs (95\% Cls) & 1.00 & 1.37 (1.24 to 1.51$)$ & 1.87 (1.77 to 1.98$)$ & 3.73 (3.26 to 4.27 ) \\
\hline
\end{tabular}

Multivariable model was adjusted for sex (men or women); level of education (no formal school, primary school, middle school, high school, college, or university or higher); marital status (married, widowed, divorced or separated, or never married); alcohol consumption (less than weekly drinker, weekly drinker, daily drinker with an intake of $<15,15-29,30-59$ or $\geq 60 \mathrm{~g} /$ day); smoking status (non-smoker, former smoker having quit smoking $\geq 5$ or $<5$ years previously or current smoker smoked $<15,15-24$ or $\geq 25$ cigarettes or equivalents per day); physical activity (MET-hours/day); intake frequencies of red meat, fresh fruit and vegetables (daily, 4-6 days/week, 1-3 days/week, monthly, or rarely or never) and family history of heart attack, stroke or cancer (presence or absence, only adjusted for in corresponding analysis of cause-specific mortality); prevalent diabetes and hypertension (presence or absence); body mass index $\left(\mathrm{kg} / \mathrm{m}^{2}\right)$ and waist- hip ratio.

${ }^{*} \mathrm{P}$ values $<0.05$ but not less than the significance level after Bonferroni correction ( 0.05 divided by 10 primary outcomes, ie, 0.005$)$. All other HRs whose $95 \%$ $\mathrm{Cl}$ did not include the null value were statistically significant after Bonferroni correction $(p<0.005)$.

HBsAg, hepatitis B surface antigen; PYs, person-years. 


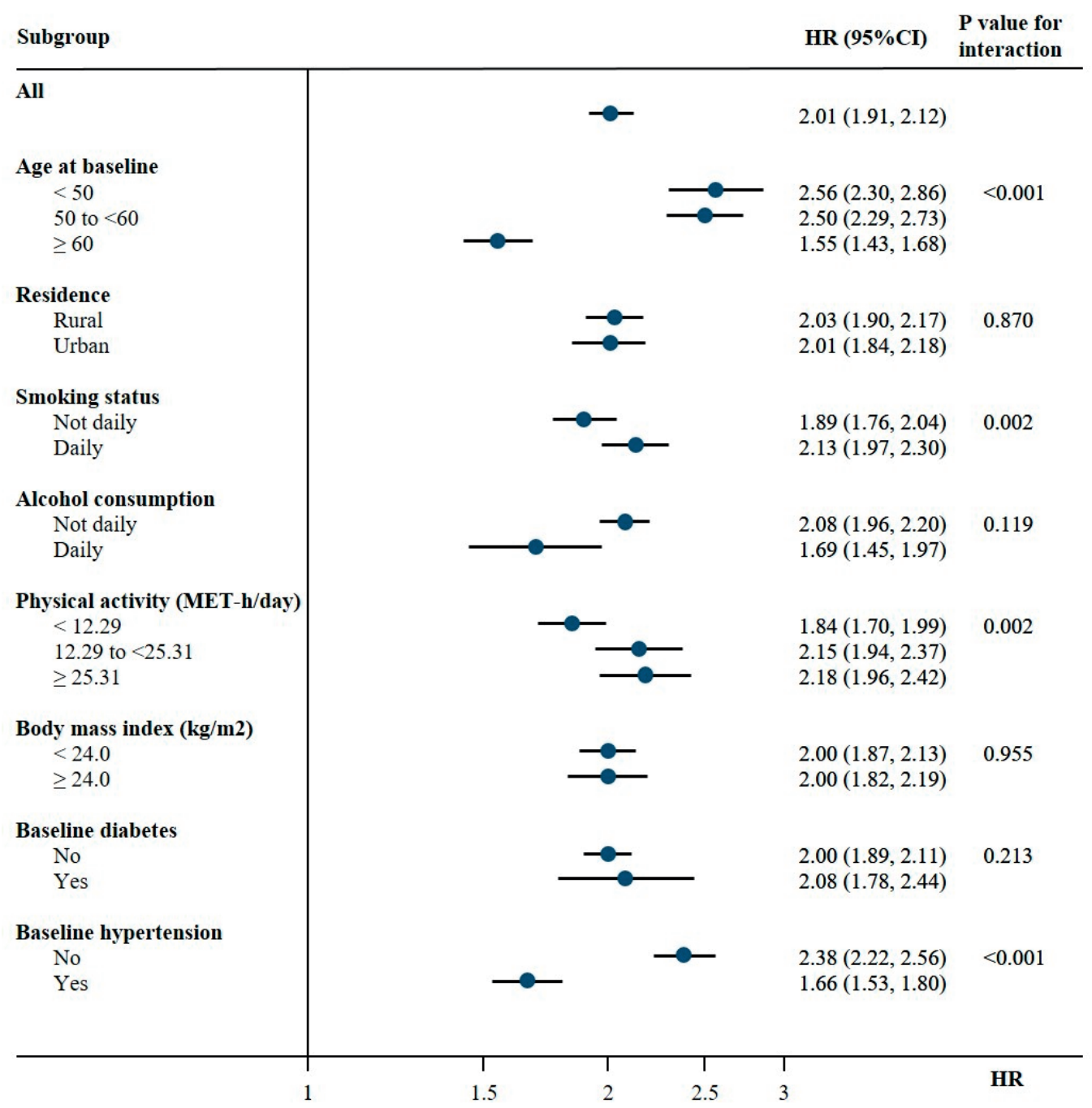

Figure 1 Subgroup analysis of the association between HBsAg status and total mortality according to potential baseline risk factors. HRs and $95 \% \mathrm{Cls}$ are for comparison of HBsAg-positive with HBsAg-negative participants. Horizontal lines represent 95\% Cls. Multivariable model was adjusted for sex; level of education; marital status; alcohol consumption; smoking status; physical activity; intake frequencies of red meat, fresh fruits and vegetables; body mass index; waist- hip ratio; prevalent diabetes and hypertension at baseline. HBsAg, hepatitis B surface antigen.

with HBsAg positivity was also seen for cause-specific death, except for those from cerebral infarction (see online supplementary file 5).

Further separation of participants according to the presence of chronic hepatitis or cirrhosis showed that HBsAg-positive participants with chronic hepatitis or cirrhosis had the greatest risk of death $(\mathrm{HR}=3.73,95 \% \mathrm{CI}$ : 3.26 to 4.27 ) (table 3). The respective HRs (95\% CIs) were 4.22 (3.62 to 4.92 ) in men and 2.55 (1.89 to 3.44) in women (see online supplementary files 6 and 7 ; $\mathrm{p}_{\text {interac- }}$ tion $<0.001)$. Similar trends were also noted in associations with death due to liver cancer, digestive diseases and infections. Sex difference in the above associations, that is, greater HRs in men than in women, was only observed for liver cancer and infections $\left(\mathrm{p}_{\text {interaction }}<0.001\right)$.

In the subgroup analyses by predefined baseline characteristics, the association between HBsAg status and total mortality was generally similar across subgroups defined by rural/urban residence, alcohol consumption, BMI and prevalent diabetes at baseline. The positive association between HBsAg status and risk of death was stronger in participants younger than 50 years, smokers, physically active participants or non-hypertensive participants (all $\mathrm{p}_{\text {interaction }}<0.01$ ) (figure 1 and see online supplementary file 8$)$.

\section{DISCUSSION}

In this large prospective cohort of 0.5 million Chinese, we found that the risk of death for participants with chronic HBV infection was twice that of those without chronic HBV infection. Median age at death was 7.7 years younger among participants with $\mathrm{HBV}$ infection than those without. Besides the significantly increased risk of death from liver cancer, infections and digestive diseases associated with chronic HBV infection, we first observed a $38 \%$ and $31 \%$ increased risk of death due to intracerebral haemorrhage and ischaemic heart diseases, respectively, 
in both men and women who lived with chronic HBV infection.

The present study was in line with previous findings from several retrospective register studies ${ }^{10-14}$ conducted in high-income countries and few prospective studies ${ }^{17} 18$ in Chinese population, in which HBV infection was associated with an increased risk of total mortality, ${ }^{10-14} 1718$ moderately increased death risk due to infections ${ }^{10-13}$ and digestive diseases ${ }^{11-14} 17$ and enormously increased death risk due to liver cancer. ${ }^{10-141718}$

The association between HBV infection and cardiovascular diseases remained equivocal. Studies conducted in Western populations have examined the association of HBV infection with death risk due to a composite circulatory endpoint (coded by ICD-9 390-459, or ICD-10 I00-I99) and got mixed results: null, ${ }^{141622}$ positive $^{1012}$ and negative ${ }^{911} 13$ results have all been reported. In the REVEAL-HBV cohort of 22,472 Chinese in Taiwan, no statistically significant association was observed between HBsAg positivity and death due to ischaemic heart diseases $(\mathrm{HR}=0.98,95 \% \mathrm{CI}: 0.82$ to 1.17$)$ and stroke $(\mathrm{HR}=0.86$, 95\% CI: 0.79 to 1.05 ) over a period of 15 years. ${ }^{16}$ Another cohort of 83754 Chinese in Haimen city of Jiangsu province found an increased risk of stroke death in HBsAg-positive men (HR=1.4, 95\% CI: 1.1 to 1.8 ) but not in women ( $\mathrm{HR}=1.3,95 \% \mathrm{CI}: 0.7$ to 2.2$)$ during 10 years of follow-up. ${ }^{18}$ Only one study, involving 521421 Korean men followed up from 1990 to 2001, examined the association between HBsAg seropositivity and the risk of incident stroke subtypes. ${ }^{23}$ It showed that HBsAg-positive men had an increased risk of intracerebral haemorrhage and decreased risks of cerebral infarction and myocardial infarction, with HRs (95\% CI) of 1.33 (1.51 to 1.52), 0.79 (0.68 to 0.90$)$ and 0.74 (0.62 to 0.87$)$, respectively. In the present study, we associated HBsAg positivity with an increased risk of dying from intracerebral haemorrhage and ischaemic heart diseases in both men and women, but not from cerebral infarction. It is possible that the relatively limited number of deaths from cerebral infarction may result in the null finding in our study.

The pathogenesis of $\mathrm{HBV}$-associated cardiovascular diseases remains unclear. A previous study found lower levels of clotting factors II and VII and fibrinogen in HBsAg-positive participants compared with HBsAgnegative ones. ${ }^{24}$ Chen et al ${ }^{18}$ suggested that decreased coagulation status due to liver cirrhosis may explain the increased haemorrhagic strokes in participants with chronic HBV infection. In the present study, the risk estimates of death due to intracerebral haemorrhage did not change materially after adjusting for the presence of chronic hepatitis or cirrhosis at baseline, suggesting that advanced liver damage might not be the causal intermediate between HBV infection and intracerebral haemorrhage. HBV-induced proinflammatory effect $^{25}$ and steatosis ${ }^{26}$ further leading to endothelial dysfunction and acceleration of atherosclerosis, may play an essential role in the development of cardiovascular diseases. The individual pathophysiological mechanisms that link the HBV infection and different cardiovascular endpoints still warrant further elucidation.

There was no association between chronic HBV infection and risk of death from respiratory diseases in the present study. Previous studies have reported divergent findings. Most of these studies compared participants with HBV infection identified through the national or regional notifiable disease surveillance system or blood donation system to the general population as the comparison group, without adjustment for any potential confounders, such as smoking. ${ }^{10-13} 18$

No previous study investigated potential interactions of HBV infection with established risk factors of several lifestyle factors and prevalent diseases on death. In the present population, we found that the association of chronic HBV infection with total mortality was stronger in smokers than in non-smokers. Smoking is an established risk factor for various diseases and death. ${ }^{27}$ The adverse impact of chronic HBV infection on death might be exacerbated by smoking-induced hepatic damage ${ }^{28}$ and systemic immune dysfunction. ${ }^{29}$ The mechanisms for their interaction still warrant further investigation. The stronger association of HBV infection with all-cause and liver cancer mortality in men than in women might be partially explained by the significantly different prevalence of smoking between sex in the present Chinese population. Also, it has been shown that men are more likely to develop liver steatosis ${ }^{30}$ and have a lower HBV clearance than women. ${ }^{31}$ Older, physically inactiveor hypertensive participants already had a high risk of death, leading to a modestly added deleterious effect by HBV infection on the relative scale.

The large sample size and extended follow-up period allowed the present study to produce reliable risk estimates for total and various cause-specific mortality. Also, this large Chinese population offered us an opportunity to examine the association between chronic HBV infection and risk of stroke subtypes, especially for haemorrhagic stroke, which is relatively uncommon in Western populations. Other strengths of the present study include a prospective cohort design, completeness of follow-up, the inclusion of a geographically spread Chinese population living in urban and rural areas and careful adjustment for potential confounders.

This study acknowledges some limitations. Considering the feasibility of conducting a large-scale population study, we used an on-site HBsAg rapid test that is convenient and repeatable but with low sensitivity for the lower seroHBsAg level. Misclassification of HBV infection status was inevitable. However, we observed a similar distribution of HBV infection in our CKB cohort with a former report ${ }^{32}$ (younger or urban participants or men were prone to a higher HBsAg-positive rate). Meanwhile, measurement errors in the prospective study may be non-differential on subsequent disease status and tend to attenuate the association towards the null. In addition, the HBsAg status was tested once at baseline, that might misclassify some participants with previous acute HBV infection. Lack of 
information about treatment precluded us from investigating the impact of treatment. In addition, we did not collect information about viral load, HBV DNA and HBeAg, which limited our ability to examine the association between HBV infection of different serological marker pattern and mortality. Some occult HBV infection participants (HBsAg negative but with measurable HBV DNA) could be missed due to the lack of HBV DNA detection. However, the prevalence of occult HBV infection is low in China, with $<0.2 \%$ of blood donors. ${ }^{33}$

In this large prospective study of the Chinese population, we found that participants with chronic HBV infection had an increased risk of death, with an especially high risk of death from liver cancer, digestive diseases and infections, and a modest but non-negligible risk of death from intracerebral haemorrhage and ischaemic heart diseases. Chronic hepatitis or liver cirrhosis patients living with HBV infection should be treated with vigour. Further studies would be essential to investigate the potential impact of the new-generation treatment. It also highlights the importance of health advice on quitting smoking, and early screening for major chronic diseases in people with chronic HBV infection. The successful implementation of $\mathrm{HBV}$ vaccinations for newborns since $1992^{34}$ in China will help prevent adult mortality associated with chronic $\mathrm{HBV}$ infection in the future.

\section{Author affiliations}

${ }^{1}$ Epidemiology and Biostatistics, School of Public Health, Peking University, Beijing, China

${ }^{2}$ Chinese Academy of Medical Sciences, Beijing, China

${ }^{3}$ Clinical Trial Service Unit \& Epidemiological Studies Unit (CTSU), Nuffield

Department of Population Health, University of Oxford, Oxford, UK

${ }^{4}$ Wuzhong Center for Disease Control \& Prevention, Suzhou, China

${ }^{5}$ Nangang Center for Disease Control \& Prevention, Harbin, China

${ }^{6}$ China National Center for Food Safety Risk Assessment, Beijing, China

${ }^{7}$ Peking University Institute of Environmental Medicine, Peking University, Beijing, China

Acknowledgements The most important acknowledgement is to the participants in the study and the members of the survey teams in each of the 10 regional centres, as well as to the project development and management teams based at Beijing, 0xford and the 10 regional centres.

Collaborators International Steering Committee: JC, ZC (PI), Rory Collins, LL (PI), Richard Peto. International Co-ordinating Centre, Oxford: Daniel Avery, Ruth Boxall, Derrick Bennett, Yumei Chang, YC, ZC, Robert Clarke, Huaidong Du, Simon Gilbert, Alex Hacker, Michael Holmes, Andri lona, Christiana Kartsonaki; Rene Kerosi, Ling Kong, Om Kurmi, Garry Lancaster, Sarah Lewington, Kuang Lin, John McDonnell, Winnie Mei, Iona Millwood, Qunhua Nie, Jayakrishnan Radhakrishnan, Sajjad Rafiq, Paul Ryder, Sam Sansome, Dan Schmidt, Paul Sherliker, Rajani Sohoni, lain Turnbull, Robin Walters, Jenny Wang, Lin Wang, LY, Xiaoming Yang. National Co-ordinating Centre, Beijing: ZB, Ge Chen, YG, Can Hou, JLv, Pei Pei, Shuzhen Qu, Yunlong Tan, CY. 10 Regional Co-ordinating Centres: Qingdao CDC: Zengchang Pang, Ruqin Gao, Shaojie Wang, Yongmei Liu, Ranran Du, Yajing Zang, Liang Cheng, Xiaocao Tian, Hua Zhang. Licang CDC: Silu Lv, Junzheng Wang, Wei Hou. Heilongjiang Provincial CDC: Jiyuan Yin, Ge Jiang, Xue Zhou. Nangang CDC: Liqiu Yang, Hui He, Bo Yu, Yanjie Li, Huaiyi Mu, Qinai Xu, Meiling Dou, Jiaojiao Ren. Hainan Provincial CDC: Shanqing Wang, Ximin Hu, Hongmei Wang, Jinyan Chen, Yan Fu, Zhenwang Fu, Xiaohuan Wang. Meilan CDC: Min Weng, Xiangyang Zheng, Yilei Li, Huimei Li, Yanjun Wang. Jiangsu Provincial CDC: Ming Wu, Jinyi Zhou, Ran Tao, Jie Yang. Suzhou CDC: Chuanming Ni, Jun Zhang, Yihe Hu, Yan Lu, Liangcai Ma, Aiyu Tang, Shuo Zhang, JJ, JL. Guangxi Provincial CDC: Zhenzhu Tang, Naying Chen, Ying Huang. Liuzhou CDC: Mingqiang Li, Jinhuai Meng, Rong Pan, Qilian Jiang, Weiyuan Zhang, Yun Liu, Liuping Wei, Liyuan Zhou, Ningyu Chen, Hairong Guan. Sichuan Provincial
CDC: Xianping Wu, Ningmei Zhang, Xiaofang Chen, Xuefeng Tang. Pengzhou CDC: Guojin Luo, Jianguo Li, Xiaofang Chen, Xunfu Zhong, Jiaqiu Liu, Qiang Sun. Gansu Provincial CDC: Pengfei Ge, Xiaolan Ren, Caixia Dong. Maiji CDC: Hui Zhang, Enke Mao, Xiaoping Wang, Tao Wang, Xi zhang. Henan Provincial CDC: Ding Zhang, Gang Zhou, Shixian Feng, Liang Chang, Lei Fan. Huixian CDC: Yulian Gao, Tianyou He, Huarong Sun, Pan He, Chen Hu, Qiannan Lv, Xukui Zhang. Zhejiang Provincial CDC: Min Yu, Ruying Hu, Hao Wang. Tongxiang CDC: Yijian Qian, Chunmei Wang, Kaixue Xie, Lingli Chen, Yidan Zhang, Dongxia Pan. Hunan Provincial CDC: Yuelong Huang, Biyun Chen, Li Yin, Donghui Jin, Huilin Liu, Zhongxi Fu, Qiaohua Xu. Liuyang CDC: Xin Xu, Hao Zhang, Youping Xiong, Huajun Long, Xianzhi Li, Libo Zhang, Zhe Qiu.

Contributors JLv and LL conceived and designed the paper. LL, ZC and JC, as the members of CKB Steering Committee, designed and supervised the conduct of the whole study, obtained funding, and together with YG, ZB, LY, YC, JJ, JLi and ZG acquired the data. JS and RM analyzed the data. JS drafted the manuscript. JLv, LL, CY, YG, ZB, RM, LY, YC, JJ, JLi, ZG, JC and ZC contributed to the interpretation of the results and critical revision of the manuscript for important intellectual content. All authors contributed to and approved the final manuscript.

Funding This work was supported by grants (2016YFC0900500, 2016YFC0900501, 2016YFC0900504 and 2016YFC1303904) from the National Key R\&D Program of China. The CKB baseline survey and the first re-survey were supported by a grant from the Kadoorie Charitable Foundation in Hong Kong. The long-term follow-up is supported by grants from the UK Wellcome Trust (202922/Z/16/Z, 088158/Z/09/Z and 104085/Z/14/Z), National Natural Science Foundation of China $(81390540,81390544$ and 81390541$)$ and Chinese Ministry of Science and Technology (2011BAI09B01).

Competing interests None declared.

\section{Patient consent for publication Not required.}

Ethics approval Central ethics approvals were obtained from the Oxford Tropical Research Ethics Committee, University of Oxford (UK) and the Chinese Center for Disease Control and Prevention (Beijing, China). In addition, approvals were obtained from institutional research boards at the local Center for Disease Control and Prevention in the 10 regions.

Provenance and peer review Not commissioned; externally peer reviewed.

Data sharing statement Details of how to access China Kadoorie Biobank data and details of the data release schedule are available from www.ckbiobank.org/ site/Data+Access

Open access This is an open access article distributed in accordance with the Creative Commons Attribution Non Commercial (CC BY-NC 4.0) license, which permits others to distribute, remix, adapt, build upon this work non-commercially, and license their derivative works on different terms, provided the original work is properly cited, appropriate credit is given, any changes made indicated, and the use is non-commercial. See: http://creativecommons.org/licenses/by-nc/4.0/.

\section{REFERENCES}

1. Seto W-K, Lo Y-R, Pawlotsky J-M, et al. Chronic hepatitis B virus infection. Lancet 2018;392:2313-24.

2. Hutin $\mathrm{Y}$, Nasrullah M, Easterbrook P, et al. Access to treatment for hepatitis B virus infection - Worldwide, 2016. MMWR Morb Mortal Wkly Rep 2018;67:773-7.

3. World Health Organization. Global Health Estimates 2015: Deaths by cause, age, sex, by country and by region, 2000-2015. http://www. who.int/healthinfo/global_burden_disease/estimates/en/index1.html (accessed 20 April 2018).

4. Liu J, Zhang S, Wang Q, et al. Seroepidemiology of hepatitis B virus infection in 2 million men aged 21-49 years in rural China: a population-based, cross-sectional study. Lancet Infect Dis 2016;16:80-6.

5. Schweitzer A, Horn J, Mikolajczyk RT, et al. Estimations of worldwide prevalence of chronic hepatitis $B$ virus infection: a systematic review of data published between 1965 and 2013. Lancet 2015;386:1546-55.

6. Polaris Observatory Collaborators. Global prevalence, treatment, and prevention of hepatitis B virus infection in 2016: a modelling study. Lancet Gastroenterol Hepatol 2018;3:383-403.

7. Beasley RP, Hwang LY, Lin CC, et al. Hepatocellular carcinoma and hepatitis B virus. A prospective study of 22707 men in Taiwan. Lancet 1981;2:1129-33.

8. Fattovich G, Brollo L, Giustina G, et al. Natural history and prognostic factors for chronic hepatitis type B. Gut 1991;32:294-8. 
9. Crook PD, Jones ME, Hall AJ. Mortality of hepatitis B surface antigen-positive blood donors in England and Wales. Int J Epidemiol 2003;32:118-24.

10. Ribes J, Clèries R, Rubió $A$, et al. Cofactors associated with liver disease mortality in an HBsAg-positive Mediterranean cohort: 20 years of follow-up. Int J Cancer 2006;119:687-94.

11. Amin J, Law MG, Bartlett M, et al. Causes of death after diagnosis of hepatitis $B$ or hepatitis $C$ infection: a large community-based linkage study. Lancet 2006;368:938-45.

12. Duberg AS, Törner A, Davidsdóttir L, et al. Cause of death in individuals with chronic HBV and/or HCV infection, a nationwide community-based register study. J Viral Hepat 2008;15:538-50.

13. Walter SR, Thein $\mathrm{HH}$, Amin J, et al. Trends in mortality after diagnosis of hepatitis B or C infection: 1992-2006. J Hepatol 2011;54:879-86.

14. Montuclard C, Hamza S, Rollot F, et al. Causes of death in people with chronic HBV infection: a population-based cohort study. $J$ Hepatol 2015;62:1265-71.

15. Fwu CW, Chien YC, Nelson KE, et al. Mortality after chronic hepatitis $B$ virus infection: a linkage study involving 2 million parous women from Taiwan. J Infect Dis 2010;201:1016-23.

16. Wang $\mathrm{CH}$, Chen $\mathrm{CJ}$, Lee $\mathrm{MH}$, et al. Chronic hepatitis $\mathrm{B}$ infection and risk of atherosclerosis-related mortality: a 17-year followup study based on 22,472 residents in Taiwan. Atherosclerosis 2010;211:624-9.

17. lloeje UH, Yang HI, Jen CL, et al. Risk and predictors of mortality associated with chronic hepatitis B infection. Clin Gastroenterol Hepatol 2007;5:921-31.

18. Chen G, Lin W, Shen F, et al. Chronic hepatitis B virus infection and mortality from non-liver causes: results from the Haimen City cohort study. Int J Epidemiol 2005;34:132-7.

19. Chen Z, Lee L, Chen J, et al. Cohort profile: the Kadoorie Study of Chronic Disease in China (KSCDC). Int J Epidemiol 2005;34:1243-9.

20. Chen Z, Chen J, Collins R, et al. China Kadoorie Biobank of 0.5 million people: survey methods, baseline characteristics and longterm follow-up. Int J Epidemiol 2011;40:1652-66.

21. Yang G, Hu J, Rao KQ, et al. Mortality registration and surveillance in China: History, current situation and challenges. Popul Health Metr 2005;3:3.

22. Katoonizadeh A, Ghoroghi S, Sharafkhah M, et al. Chronic hepatitis $B$ infection is not associated with increased risk of vascular mortality while having an association with metabolic syndrome. J Med Virol 2016;88:1230-7.

23. Sung J, Song YM, Choi YH, et al. Hepatitis B virus seropositivity and the risk of stroke and myocardial infarction. Stroke 2007;38:1436-41.

24. Meade TW, Stirling Y, Thompson SG, et al. Carriers of hepatitis B surface antigen: possible association between low levels of clotting factors and protection against ischaemic heart disease. Thromb Res 1987;45:709-13.

25. Epstein SE, Zhu J, Najafi AH, et al. Insights into the role of infection in atherogenesis and in plaque rupture. Circulation 2009;119:3133-41.

26. Chaabane NB, Loghmari $\mathrm{H}$, Melki W, et al. Chronic viral hepatitis and kidney failure. Presse Med 2008;37:665-78.

27. U.S. Department of Health and Human Services. The health consequences of smoking - 50 years of progress: a report of the surgeon general. Atlanta, GA: U.S. Department of Health and Human Services, Centers for Disease Control and Prevention, National Center for Chronic Disease Prevention and Health Promotion, Office on Smoking and Health, 2014.

28. Liu Y, Dai M, Bi Y, et al. Active smoking, passive smoking, and risk of nonalcoholic fatty liver disease (NAFLD): a population-based study in China. J Epidemiol 2013;23:115-21.

29. Shiels MS, Katki HA, Freedman ND, et al. Cigarette smoking and variations in systemic immune and inflammation markers. J Natl Cancer Inst 2014;106.

30. Machado MV, Oliveira AG, Cortez-Pinto H. Hepatic steatosis in hepatitis $B$ virus infected patients: meta-analysis of risk factors and comparison with hepatitis $C$ infected patients. J Gastroenterol Hepatol 2011;26:1361-7.

31. London WT, Drew JS. Sex differences in response to hepatitis B infection among patients receiving chronic dialysis treatment. Proc Natl Acad Sci U S A 1977;74:2561-3.

32. Huang P, Zhu LG, Zhu YF, et al. Seroepidemiology of hepatitis B virus infection and impact of vaccination. World J Gastroenterol 2015;21:7842-50.

33. Zheng $X, Y e X$, Zhang $L$, et al. Characterization of occult hepatitis $B$ virus infection from blood donors in China. J Clin Microbiol 2011;49:1730-7.

34. Liao X, Liang Z. Strategy vaccination against Hepatitis B in China. Hum Vaccin Immunother 2015;11:1534-9. 